\title{
Thematic analysis of acceptability and fidelity of engagement for behaviour change interventions: The Let's Move It intervention interview study
}

\author{
Minttu Palsola '* (D), Elina Renko' (D), Katri Kostamo' (D), \\ Fabiana Lorencatto $^{2}$ (D) and Nelli Hankonen' (D) \\ 'Faculty of Social Sciences, University of Helsinki, Finland \\ ${ }^{2}$ Centre for Behaviour Change, University College London, UK
}

Objectives. Intervention participants' responses to and engagement with interventions are a key intermediate step between interventions and intended outcomes. The aim of this study was to qualitatively investigate crucial aspects of engagement, namely acceptability (experienced cognitive and emotional responses to the intervention), receipt (comprehension of intervention content), and skill enactment (skill performance in target settings), within the Let's Move It, a multi-component school-based physical activity intervention.

Design. A longitudinal qualitative study embedded in a cluster-randomized trial, with individual interviews of purposefully sampled intervention participants immediately postintervention $(n=21)$ and at 14 months $(n=14)$.

Methods. Semi-structured interviews were analysed using thematic analysis. Abductive coding process was taken to identify categories for themes.

Results. The analysis resulted in 12 themes and 18 subthemes. Overall, participants reported perceived effectiveness of and affective attitude towards the intervention (acceptability) and understood the main messages and skills (receipt). For example, findings indicated comprehension of the non-judgemental nature and choice-providing messages of the intervention underpinned by self-determination theory. Despite reporting understanding how and why to perform the skills, not using them was a highlighted theme (skill enactment), particularly for self-regulatory techniques such as planning. Friends' role as key self-motivation technique was a prevalent theme. In the within-individual analysis, three different engager types were identified: positive, ambivalent, and negative.

Conclusion. Identifying misunderstandings and difficulties in skill acquisition can help interpret main trial outcomes and inform further intervention optimization. This study provides an example of how to use thematic analysis to assess acceptability, receipt, and enactment in interventions.

\footnotetext{
This is an open access article under the terms of the Creative Commons Attribution License, which permits use, distribution and
} reproduction in any medium, provided the original work is properly cited.

*Correspondence should be addressed to Minttu Palsola, Unioninkatu 37, 00170 Helsinki, Finland (email: minttu.palsola@ helsinki.fi). 


\section{Statement of contribution}

\section{What is already known on this subject?}

- Participant engagement with interventions is crucial for effectiveness.

- Intervention acceptability, receipt, and skill enactment are key elements of engagement.

- Few studies have addressed these with qualitative methods despite added value for trial interpretation.

\section{What does this study add?}

- Intervention was well accepted and comprehension was mixed.

- Unintended comprehension indicated misunderstanding of key theory-based skills.

- Thematic analysis of participant interviews is a feasible way to study quality of engagement.

Randomized trials do not explain why or how things work, only whether they work (Deaton \& Cartwright, 2018). To investigate the why and how, conducting process evaluations alongside outcome evaluations and RCTs is beneficial. These evaluations can take many forms and explore different elements. The Medical Research Council's process evaluation guidance (Moore et al., 2015) outlines the importance of exploring intervention context, engagement, and implementation. This can help more accurately interpret trial outcomes, support intervention replication, scalability, and optimization, yet process evaluations are often an overlooked element. This paper aims to illustrate a methodological and analytical approach to conducting a process evaluation of a schoolbased physical activity intervention and demonstrate how qualitative investigations embedded in trials can shed light onto the expected change mechanisms.

\section{Acceptability, receipt, and skill enactment in the Let's Move It intervention context}

Youth worldwide engage in too little physical activity (PA) for their health and well-being (Elgar et al., 2015). School-based interventions have been developed to increase PA and decrease sedentary behaviour (SB; Hynynen et al., 2016), some of which operate via indirect mechanisms, that is, altering physical environments to increase opportunities for PA, while others target more conscious and reflective mechanisms by aiming to alter the PA-related beliefs and skills.

In order for interventions to be effective, they need to be accepted by participants and conducted with high fidelity. Fidelity refers to the methodological strategies used to monitor and enhance the reliability and validity of behavioural interventions (Bellg et al., 2004). However, interventions can be delivered with perfect fidelity, but not work as intended if participants do not accept and engage with them. We draw on frameworks of acceptability (Sekhon, Cartwright, \& Francis, 2017) and fidelity (Bellg et al., 2004) to discuss participant engagement. They are both multi-component constructs relevant at the intervention designer, provider, and recipient levels (Bellg et al., 2004; Sekhon et al., 2017), and thus intertwined. We use engagement (Walton, Spector, Tombor, \& Michie, 2017) as an umbrella term for receipt and skill enactment (Bellg et al., 2004) and see acceptability as an interrelated, yet distinguishable, construct (Sekhon et al., 2017).

Acceptability, that is, experienced cognitive and emotional responses to the intervention, is a prerequisite (but not a sufficient condition) for a successful intervention (Sekhon et al., 2017). Acceptability reflects the extent to which the intervention is considered appropriate, and can be seen as a predictor of intention to engage in the intervention (Sekhon et al., 2017). Acceptability consists of affective attitude (feelings 
about taking part in an intervention), burden (effort required to participate in the intervention), perceived effectiveness (likelihood for the intervention to achieve its purpose), ethicality (fit with value system), intervention coherence (understanding the intervention and how it works), opportunity costs (what must be given up to engage in an intervention), and self-efficacy (confidence that the behaviours required to participate in the intervention can be performed; Sekhon et al., 2017).

Receipt refers to the participant side of fidelity, that is, comprehension of intervention and performance of the cognitive and behavioural skills taught in the intervention (Bellg et al., 2004). It is a precondition for enactment, that is, the intervention participants using the skills delivered in the intervention in the target setting, and is thus an important part of possible impact mechanisms (Moore et al., 2015). Receipt and enactment may resemble treatment adherence, but for behaviour change studies, these are distinguishable concepts. Receipt relates to skill use in the intervention setting (e.g., learning goalsetting), and enactment relates to skill use outside the intervention (e.g., planning for PA sessions), whereas adherence relates to the definitive tasks in the health behaviour (e.g., increasing PA; Bellg et al., 2004).

The importance of assessing concepts of engagement for complex behavioural interventions has been increasingly emphasized (Bellg et al., 2004; Sekhon et al., 2017; Walton et al., 2017). However, acceptability assessments have lacked common guidelines (Sekhon et al., 2017) and most fidelity assessments have focused on provider delivery, that is, the extent to which the intervention was delivered as intended (Rixon et al., 2016; Walton et al., 2017). According to a recent review of health interventions (Rixon et al., 2016), only $19.6 \%$ of the identified reports on fidelity were found to address receipt, usually not within main trials nor in relation to both understanding and performing the skills. Furthermore, only a handful of receipt studies have relied on qualitative interview data (Rixon et al., 2016), and most engagement assessments on self-reports or intervention records (Walton et al., 2017).

Lack of acceptability, receipt, and enactment analyses is a clear limitation in the current literature. We acknowledge that these concepts of engagement are complex, interrelated phenomena, and thus employ qualitative methods to explore perspectives surrounding the complexity in order to provide a rich, contextualized understanding of engagement and the expected change mechanisms. This can help identify potential weak links in the assumed causal model (Moore et al., 2015), and thereby illuminate quantitative findings of effectiveness evaluations and advance intervention development.

We assess acceptability, receipt, and enactment as part of a comprehensive evaluation strategy for a cluster-randomized trial evaluating effectiveness and processes of the schoolbased Let's Move It (LMI) intervention. The main trial included six schools (Hankonen et al., 2016) with 528 control and 638 intervention arm participants at baseline (Heino et al., 2019). The student intervention component was formulated based on selfdetermination theory (Deci \& Ryan, 2000), reasoned action approach (Fishbein \& Ajzen, 2010), and self-regulation theories (Carver \& Scheier, 1982) providing participants with behaviour change techniques (BCTs; Michie et al., 2013) and PA-promoting choice architecture (Hankonen et al., 2016). It included (1) group sessions (S1-S6) and a poster campaign (targeting autonomous PA motivation, and self-regulation skills such as planning, SMART goal-setting, self-monitoring, and self-motivation), (2) active classrooms (e.g., gym balls and standing desks, teachers trained to give activity breaks), and (3) enhanced PA opportunities (Hankonen et al., 2016). Apparent across sessions, materials, and core learning outcomes of the LMI intervention were LMI's main principles: (1) Any activity is better than nothing, (2) your own choice: whether you are active, and how (3) 
we are all entitled to activity, (4) know what moves you, (5) the goal is well-being, not a fatless body, and (6) sitting sucks. The full LMI intervention content and recruitment process are described elsewhere (Hankonen, Absetz, \& Araújo-Soares, in press). In this context, indications of acceptability include, for example, approval of the intervention's principles; indications of receipt, for example, describing goal-setting; and indications of enactment, for example, describing self-monitoring PA in one's free time.

\section{Research aims}

Our aim is to demonstrate the methods and analytical approach taken to access acceptability, receipt, and skill enactment as a part of the LMI process evaluation. Specifically, we investigated how participants describe their:

1. Views of the intervention as a whole (acceptability)

2. Understanding of the intervention content, and how it aligns with the intended intervention (receipt)

3. Skill use outside the intervention setting, and how it aligns with the intended intervention (enactment; Figure 1).

\section{Method}

\section{Study design and setting}

Data were collected in the context of the LMI intervention. Interviews were conducted after the intensive intervention at 6-8 weeks post-baseline (I-1), and 14 months after baseline (I-2).

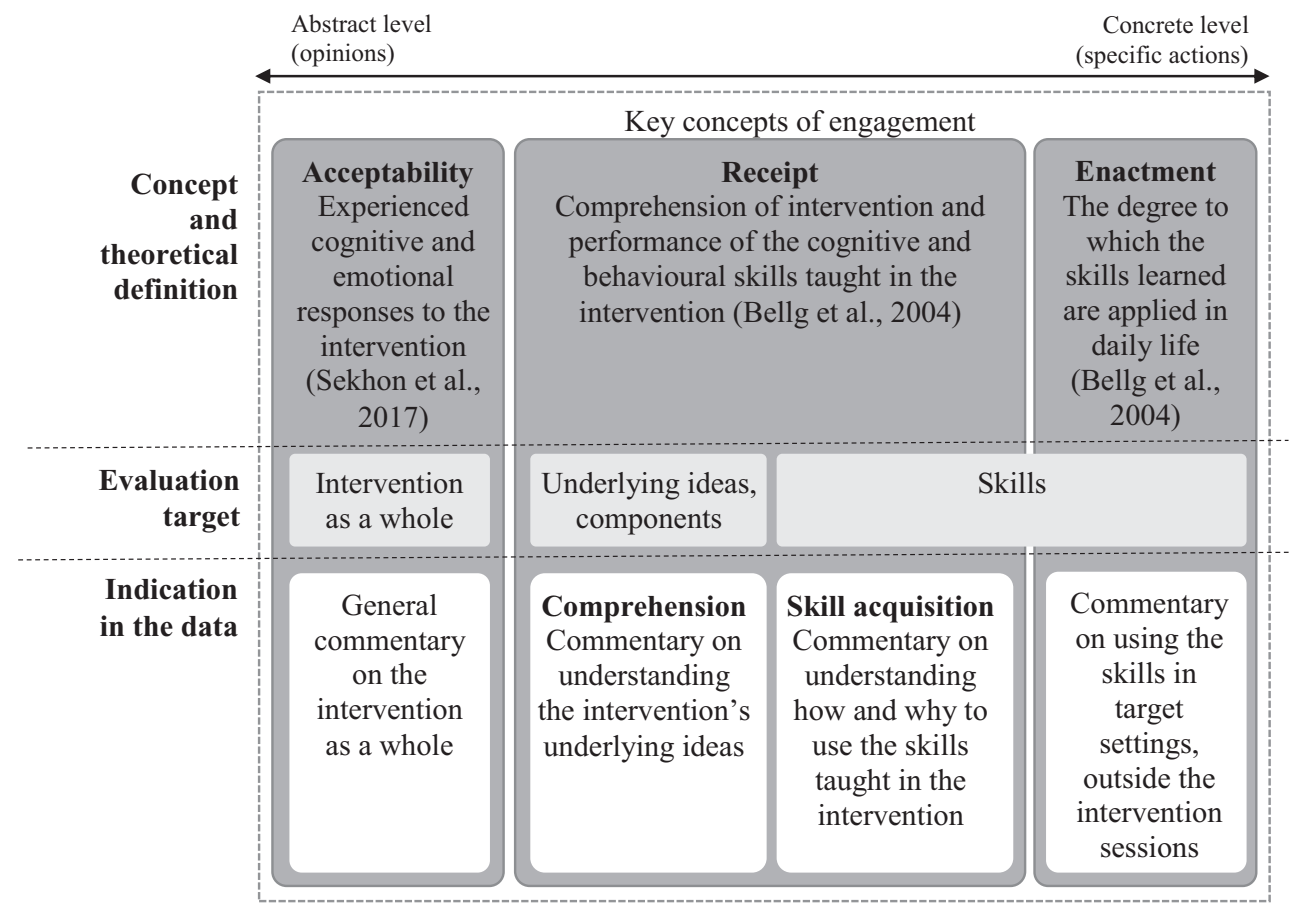

Figure I. The scope of the LMI engagement assessment. 


\section{Study participants}

For the qualitative evaluation, we invited a subsample of participants $(n=34)$ using purposeful sampling. Previously low-to-moderately active LMI participants were identified based on self-reports at baseline and invited to participate in the interviews. Of the 21 interviewed participants, 14 attended both I-1 and I-2 (Table 1). Other criteria for interviewing were basic vocational degree, signed consent for the study, age of 1519 years, minim. 4/5 self-assessed skills in Finnish language, no background in competitive sports, and at least 4 LMI sessions attended. This study excludes control participant interviews, reported elsewhere (Kostamo, Jallinoja, et al., 2019).

\section{Procedure}

Research assistants contacted participants face-to-face for both interviews, and if not met at school, via phone. These participants were given study information sheets and they signed a consent for participation and audio recording. As an incentive, participants were offered a movie voucher. The ethical committee of the Hospital District of Helsinki and Uusimaa (367/13/03/03/2014) has reviewed the study procedures.

The interviews were conducted one-on-one and face-to-face, and lasted 24-80 $\mathrm{min}$. Four research assistants (independent from intervention delivery) were trained to follow an interview strategy of showing interest, welcoming all answers, engaging participants in conversation, and reflective listening. A semi-structured topic guide (Files S4 and S5) consisting of four topics was used both at I-1 and I-2 (Figure 2). The topic guide was developed based on the programme theory to invite commentary around its key elements and uncertainties. This analysis focuses on topics 2 and 4 , best inviting commentary around concepts of engagement (Figure 3). Audio recordings were transcribed verbatim.

\section{Analysis}

Thematic analysis with essentialist approach was used as the analytical method; that is, participants' responses were assumed to reflect their experiences, meanings, and reality

Table I. Distribution of participants in regard to gender, school, and educational track

\begin{tabular}{lll}
\hline Participants in intervention schools & \multicolumn{1}{c}{ I-I } & \multicolumn{1}{c}{ I-2 } \\
\hline Practical nurse students & 14 ( I 2 females, 2 males $)$ & 10 (9 females, I male) \\
Hotel, restaurant, and catering students & 7 (5 females, 2 males $)$ & 4 (3 females I male) \\
In total & 21 (I7 females, 4 males $)$ & 14 (I2 females, 2 males) \\
\hline
\end{tabular}

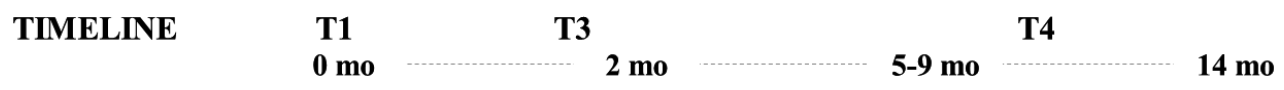

\begin{tabular}{|l|lll|}
\hline Student sessions & S1 S2 S3 S4 S5 S6 & B \\
\hline Other components & $\begin{array}{l}\text { Classroom sitting reduction intervention, } \\
\text { poster campaign, PA possibility enhancement }\end{array}$ \\
\hline Interviews & & I-1 & I-2 \\
\hline
\end{tabular}

$\mathrm{S}=$ group session, $\mathrm{B}=$ booster session $\mathrm{I}=$ interview

Figure 2. Diagram summarizing main components and timelines of LMI. 


\section{TOPIC 1: Changes in PA behavior}

Questions investigating what role PA plays in participants' lives and whether there have been any changes in it. The participants were presented with a timeline as a stimulus.

\section{TOPIC 2: Strategies to increase, maintain or manage PA}

Structured around central components of the LMI intervention theory to invite talk about the intervention and its content. Open-ended questions on self-motivation for doing PA, planning, self-monitoring, and thoughts on sitting reduction.

\section{TOPIC 3: PA-related thoughts}

Questions to tap more on the participants' relationship with PA, and whether they anticipate it to change in their near future.

\section{TOPIC 4: LMI content}

Workbook used as a prompt. Participants were asked if they found the content useful, whether or not they had considered or already used the selfregulation and self-motivation tools in the workbook, and what they thought about the sitting reduction program. No direct questions about acceptability or tests on comprehension.

\section{TOPIC 4}

(a) Perceptions on PA (prompted)

Participants were presented with four central LMI principles one by one and asked whether or not they agree with them, and why.

\section{(b) LMI content}

The participants were directly asked if they plan, use the LMI videos or recall seeing posters, and what they remember about the sessions.

Figure 3. Contents of the interview topic guides.

(Braun \& Clarke, 2006). The analysis was semantic as we were looking for explicit meanings (Patton, 1990). The process followed guidelines by Braun and Clarke (2006). For coding, we used abductive approach (Tuomi \& Sarajärvi, 2002, p. 99) as we moved back and forth between observations and theoretical generalizations (Tavory \& Timmermans, 2014). We describe the analysis as abductive, rather than deductive (theory/analyst-driven), to help the reader to understand the relationship between theory, data, and method and to be more explicit about the process (see Files S1 and S2).

The process of coding the data started without trying to fit the data into a pre-existing coding frame, that is, inductively; the focus was on general engagement with the intervention. First, the data were read as a whole to form a comprehensive understanding. Initial ideas for coding were noted down by the first author (MP). Initial codes were systematically generated with a focus on LMI programme-related comments (topic 4). All responses were thoroughly coded using word-specific codes in order to sustain the nuances at this point of analysis. A decision to include other BCT-related responses to enrich the talk was made due to participants' short answers on the intervention programme (topic 2). These data units were coded if the participants made a connection to LMI, for example, by mentioning the lessons 'maybe because we have had these 
lessons' or materials 'we have this book'. Finally, specific initial codes were generated for these topics in I-2. Next, the excerpts were coded by valence as intended/unintended.

Only after this, the concepts of acceptability, receipt, and enactment were identified as tools to analyse the data and the research questions were refined accordingly. Now the data were approached with specific questions in mind, and MP collated codes into potential themes under them. Comments regarding the general views on LMI were categorized under acceptability, comments regarding comprehension under receipt, and comments regarding skill use under enactment. While initial codes were generalized to plausible overarching themes and subthemes, specific codes were maintained not to lose the potential for other subthemes in later phases. A criterion for forming a theme was at least two occurrences in the data in at least two interviews. To sustain the focus on the participants' perceptions of the programme, codes were excluded if their relation to LMI cannot be shown, or if the interviewer seems to be pushing for an answer. All possibly relevant codes without clear overarching themes were retained at this phase and themed as 'anomalies'. The first draft of the thematic map was created and reviewed with coauthors (each member individually) in relation to the coded extracts to ensure internal homogeneity within and external heterogeneity between themes. There were no major disagreements about the validity of the themes, but some minor adjustments were made and some themes were combined under one overarching theme. Based on the review, MP reread the data set and coded all units that were missed in the earlier phases or needed regrouping. These themes were then compared to the LMI intervention content, principles and aims (Hankonen et al., in press), and coded either intended or unintended based on whether or not they reflected the intervention theory and aims.

Finally, the thematic map was refined, including final definitions and names for each theme reflecting the LMI content and theoretical frameworks. After this, each participant's themes were mapped separately in order to see differences between theme distributions.

\section{Results}

Figure 4 displays an overview of the results categorized by acceptability, receipt, and enactment. Throughout the results section, time point and participant number are indicated in brackets.

\section{Category I: Acceptability-related themes}

Four main themes fall under this category. Not all of the dimensions of the theoretical framework of acceptability (TFA; Sekhon et al., 2017) are present, as the themes were first identified based on the data and then compared to TFA. The dimensions identified were affective attitude, perceived effectiveness, self-efficacy, and intervention coherence.

Theme I. Affective attitude and perceived effectiveness of the programme and sessions Almost all participants reported perceiving the LMI sessions positively: "I am happy that she told us these things and that our class was selected, the gauges and all, because everyone learned a lot. And everyone wanted to be there. Because it made us feel, you know, good" (I-2:6). 
Table 2. Overview of findingsGreen indicates intended (=in line with intervention theory and aims), orange mixed (=partly in line with intervention theory and aims), and red unintended (=not in line with intervention theory and aims) outcomes.

\section{Intended}

\section{Unintended}

1. Affective attitude and perceived effectiveness of the program and sessions

1.1. Sessions fun

1.2. Sessions useful

3. Affective attitude towards and perceived effectiveness of the materials and activities 3.1. Workbook and activities helpful in changing PA habits

3.2. Posters as reminders and motivators

4. Affective attitude towards and intervention coherence of sitting reduction

1. Intended comprehension of the main messages

1.1. $\mathrm{PA}$ as a right and choice.

1.2. Importance of everyday PA

1.3. Goal is well-being, not a fatless body

1.4. "Sitting sucks"

2. Mixed comprehension of self-regulatory skills

2.1. Ability to use self-regulatory skills

2. Lack of intervention coherence and perceived effectiveness

1. Limited enactment of self-regulatory skills

1.3. Self-reminders as a way to increase PA

2. Mixed enactment of self-motivation techniques

2.1. Using friends for commitment and encouragement

2.3. Allowing oneself to start PA gradually
1.1. Light self-monitoring

1.2. Lack of planning

1.4. Goal-setting related to outcomes rather than

PA

2.2. Not for me.

3. LMI unintendedly perceived as a part of school

2.2. Reminding oneself of the good consequences but not for self-motivation purposes

2.4. No motivators

\section{Online materials not in use}

Theme I.I. Sessions perceived as fun

The participants described the sessions as uplifting, or said even that 'somehow the program was the best' (I-1:22).

Theme 1.2. Sessions perceived as useful

The participants mentioned gaining new information, for example, through class discussions: 'we talked about why to do PA and when and how, and I have thought about it, and it has been nice because I got tips on how to do this and that' (I-1:43). The programme was viewed motivating in engaging in PA. 'The sessions gave me motivation that it is good for you to be physically active and 
you should really do it' (I-1:27). A few also stated that 'Let's Move It got me to move more' (I-1:22).

\section{Theme 2. Lack of intervention coherence and perceived effectiveness}

The programme elicited very little negative commentary. However, some participants mentioned that there was no new information, which made the lessons boring and repetitive and the content 'self-evident' (I-1:3) and 'common sense' (I-1:33).

\section{Theme 3. Affective attitude towards and perceived effectiveness of the materials and activities}

Participants also talked about the LMI materials in an acceptability-indicating way, generally perceiving them useful and entertaining.

\section{Theme 3.I. Workbook and activities helpful in changing PA habits}

When asked about the usefulness of the intervention content in changing PA habits, many participants referred to the workbook and session activities as interesting and helpful in changing their views, increasing PA and learning from others in the classroom, thus indicating affective attitude and perceived effectiveness: '- that you get to hear what others have done. Or what they have planned or what their hobbies are and. The notebook was good' (I-1:22).

\section{Theme 3.2. Posters as reminders and motivators}

When asked if they remember seeing posters in the school, some participants indicated perceived effectiveness as they described finding the posters motivating, and reminding them of the intervention content and their PA plans: '-when I see a Let's Move It poster, it instantly pops into my head that oh yeah I have a gym day today' (I-2:5). Also, some found the posters entertaining, thus indicating affective attitude: 'The posters were funny, had a little bit of humour. It was, like, fun to read them' (I-2:6). Particularly, the messages targeting sitting reduction were recalled.

\section{Theme 4. Affective attitude towards and intervention coherence of sitting reduction}

Most participants described enjoying using the equipment in the classroom, especially the balls. Many indicated intervention coherence by discussing the rationale behind sitting reduction: "First I was like, what are these and who brought them here, but then I realized that oh yeah these are meant to help and I think it is of course for a good reason. That you reduce sitting, or like sitting still during classes. I thought it was a real positive thing" (I-1:25).

Some indicated perceived effectiveness by describing being able to concentrate better when they 'can bounce on the ball' (I-1:12) or 'stretch their necks' (I-1:20) and did not have to sit still.

Few also seemed to view teacher involvement as a condition for effectiveness of the sitting reduction. They mentioned doubting being allowed to be more active in the classroom or to use the equipment without the teachers understanding the rationale: "If you hadn't come I don't think that they would have let us, but since you came and told more it has helped. That they don't say like, put that away anymore" (I-1:24). 


\section{Category II: Receipt-related themes}

Three main themes fall under the category of intervention receipt, namely whether LMI's main messages and delivered BCTs were understood.

Theme I. Intended comprehension of the main messages

Participants' responses demonstrated understanding of LMI's main principles. The participants were not directly asked about these principles, but they spontaneously talked about them when asked what they remember about the programme or if anything had changed in their thinking. The principles 'any activity is better than nothing' and 'sitting sucks' were referenced exactly as phrased in the intervention, whereas the other principles were discussed in youth's own words.

Theme 1.1. PA as a right and choice. Often, the participants mentioned that any PA is better for well-being than none and everyone has the right to engage or not to engage in PA and can choose their own way of doing it. The data suggested that the participants generally understood these three principals as one entity. "The Let's Move It people, they showed us that you can be active in different ways, - Like, it is our option if we do it or if we don't. That they won't judge" (I-1:5).

Theme 1.2. Importance of everyday PA. A majority of participants discussed LMI opening their eyes to new opportunities and insight into the importance of incidental PA in their lives: "all the vacuuming that you do, like you walk at your home, and all the walks to school, they have opened my mind in a sense that those are important, too" (I-1:44).

These statements reflect the acceptance of 'any activity is better than nothing'. Incidental PA was promoted in all sessions by providing tips on how to integrate PA in daily activities.

Theme 1.3. Goal is well-being, not a fatless body. Let's Move It, in line with SDT as its theory base, promoted intrinsic rather than extrinsic goals for PA (Deci \& Ryan, 2000; Hankonen et al., 2016) and emphasized well-being, rather than achieving a fatless body or focusing on looks. This theme directly reflects 'the goal is well-being, not a fatless body' principle and, for example, the fitspiration media message criticism activity (Hankonen et $a l$., in press). When participants were asked about their reasons to be active they generally talked about 'A healthier life. A good feeling. A positive feeling' (I-1:4). Some mentioned weight loss or looks in passing, but they did not explicitly link these goals to the intervention content.

Theme 1.4. 'Sitting sucks'. Almost all participants discussed the importance of sitting reduction, and some reported that they had never thought about it before '-but then the Let's Move It thing was like hey, this is really not good for you' (I-1:43). However, despite agreeing with the message 'sitting sucks', a handful of participants mentioned finding sitting reduction irrelevant for them due to being already active enough or not sitting that much. This cannot be considered unintended since it cannot be reliably determined whether they indeed are sufficiently active. 
Theme 2. Mixed comprehension of self-regulatory skills

Participants were taught a selection of self-regulatory strategies, for example, SMART goalsetting (i.e., a PA goal should be specific, measurable, attainable, relevant, and timebased). They were guided to plan what, when, where, and with whom they would be physically active, and later also $w h y$ (personally relevant reasons). Self-monitoring tools were provided, for example, a paper-and-pencil diary to record PA sessions or a selfchosen mobile app (for more details, see Hankonen et al., in press). Several self-motivation techniques were also taught, for example, finding one's own reasons to be physically active and seeking social support. Commentary on understanding how and why to use the taught skills was mixed.

Theme 2.1. Ability to use self-regulatory skills. Almost all participants could generate and identify ways to self-motivate themselves to do PA, and accepted the idea of selfmotivation. Several participants discussed the usefulness of SMART. They demonstrated knowing how to use SMART, and understanding why to use planning skills: "because it might be like you think that next week I will go for a run and maybe to the gym, so you might forget but if you write down what you have to do and when and list reasons why you want to do it and all, then you will remember" (I-1:42).

The participants often talked about understanding the positive consequences of selfmonitoring and few mentioned gaining new insight into its helpfulness in tracking everyday PA.

Theme 2.2. Self-regulatory skills are not for me. The participants indicated intended comprehension on how and why to use the skills, but did not attribute the skills useful or suitable for themselves. They said, 'for some it might help in terms of PA that you have a certain rhythm' (I-1:3), but that only people with certain characteristics that they themselves lack benefited from planning. They described themselves as 'lazy with these sorts of things' (I-1:45) or '110 percent sure of being forgetful' (I-2:19), or planning 'unnatural' (I-1:4) for them.

Theme 3. LMI unintendedly perceived as a part of school

Although the participants generally understood the intervention messages as intended, a small portion referred to LMI as 'a course among others' (I-2:16), and did not consider the possibility of utilizing the materials outside the lessons unless they were assigned as homework nor perceive them useful outside the school setting. This indicated an unintended misunderstanding of the intervention developers' original purpose, 'for life, not for school only'.

\section{Category III: Skill enactment-related themes}

Commentary on skill enactment was not easily categorized as intended and unintended. Thus, most categories represent mixed enactment. Two participants discussed complete disinterest towards enacting any skills and gave not seeing PA as a part of their life as a reason for this. One said that '-when I am on my free-time I do not think about PA, I just think about the other things in my life' (I-1:25), whereas the other stated that 'PA does not interest me' (I-1:12). 
Theme I. Limited enactment of self-regulatory skills

Participants often mentioned that planning and self-monitoring require time and effort. Thus, the time spent on those meant less time to spend on something else. Only two participants demonstrated the use of self-regulation skills as an optimal logical entity of planning, self-monitoring and adjusting plans accordingly: "I take a small piece of paper where to write what to do today, like a to-do list, like for how long, I make a mark when I have done it and if I have not done it I do it before I go to bed" (I-2:1).

Some reported using non-specific planning, for example, 'I just have it in my head what to do' (I-1:4) and 'I just think about when I might have time' (I-1:5).

Theme 1.1. Light self-monitoring. One participant described intended enactment of self-monitoring: "you mark down also all the everyday PA and such, so at the end of the week you see what you have done, even if you have done something outside your plan, which is good" (I-1:42).

Some mentioned automating self-monitoring with mobile apps, whereas some discuss self-monitoring outcomes rather than the amount of PA, for example, by being able to add more weights or testing their progress whether they 'have to catch their breath when running up the stairs' (I-1.26). Progress monitoring was not emphasized in the intervention as much as behavioural self-monitoring.

Theme 1.2. Lack of planning. Students were advised to plan but not to force themselves to do PA. Yet, participants described that planning poses a risk: '-it is not fun if you just plan thoroughly and make a schedule as if it was school or work' (I-2:6). For some, 'the plan would just make it a little heavier in a way that you might end up doing nothing' (I1:6). Participants also discussed their fear of not being able to fulfil the plan: '- if you have planned something and something comes up it is very annoying' (I-1:24). Some saw no need for planning since they already had PA routines or they were not active enough to need a plan: 'these might be meant for those who have like, athletes they have a lot of different sports they do, that they can do stuff like this' (I-2:7).

Theme 1.3. Self-reminders as a way to increase PA. The LMI facilitators gave tips on how to remind oneself of the plans, but self-reminders were not a central focus of the intervention. Few participants discussed the benefits of self-reminders in increasing PA. They talked about placing PA plans in a visible spot, for example, on a wall or a door in order to 'remember that oh yeah it is a gym day and you take your equipment and go' (I2:5) or setting alarms on their phones. Some reminded themselves of their plans, others of their self-set goals: 'I have a matrix at home, displayed on my room's door, that it has all my goals and all' (I-2:6).

Theme 1.4. Goal-setting related to outcomes rather than PA. When asked about their PA-related plans or goals, the majority of participants indicated suboptimal enactment, as they gave non-specific responses reflecting outcome goal-setting, such as 'getting in shape' (I-1:5) or 'having a better physique' (I-1:45), instead of behavioural goal-setting. These responses are not in line with SMART, specifically linked to behavioural goals. 
Theme 2. Mixed enactment of self-motivation techniques

Participants discussed three self-motivation techniques promoted in LMI: using friends for commitment, reminding oneself of the good consequences of PA and graded tasks.

Theme 2.1. Using friends for commitment and encouragement. LMI encouraged participants to utilize social support by engaging friend(s) or family in their PA plans and taught strategies to actively seek social support (Hankonen et al., in press). The participants mentioned making plans with friends as a good way to commit to PA: 'make a plan together earlier then the plan is done, like let's go and then you go and can't be like alone like I don't feel like going' (I-1:10). In addition, they described having a friend as encouraging, giving extra motivation, and making PA more fun. A couple of participants linked the use of friends to the SMART planning in defining the 'what, why, where, when and with whom', albeit using it knowingly: "if I plan to go swimming with a friend we quite often do plan that wha-, like when and where, and like then you actually go with you friend, but not - like I would notice that I've used them" (I-1:21).

Using friends as motivators seemed to be especially central, even if the original plan with the friend did not work out: 'I can ask someone else to come, that, if i can go I could go with someone else and not with the one I usually go with' (I-1:10). One participant said that weekends might sometimes be inactive because he/she 'couldn't get any friend to go and do something' (I-1:21).

Theme 2.2. Reminding oneself of the good consequences but not for self-motivation purposes. The participants were guided to think about the good consequences of PA. In line with this, participants often mention reminding themselves of consequences such as feeling good and PA being worth the effort, but also that they do not actively do this for self-motivation purposes.

Theme 2.3. Allowing oneself to start PA gradually. 'Graded tasks' has been a feature of effective interventions (Hynynen et al., 2016) and help gradually develop self-efficacy. One central message in LMI was that PA is not a matter of 'all or nothing' and that 'graded tasks' are beneficial, that is, starting with lower amount and intensity, and increasing them gradually. In accordance, some participants described using graded tasks: "I started to run first five, ten minutes, caught my breath, and next time I run twenty minutes, thirty, and now it is an hour, - I learned that you don't have to be afraid - that it is not just after one night but that the achievement will come, just little by little" (I-2:5).

A couple of participants discussed allowing themselves to stop if they feel too tired: 'if you like usually do PA for let's say an hour, that if you don't feel like it do half an hour instead' (I-1:20).

Theme 2.4. No motivators. Few participants reported that they 'have not figured out what would motivate them' (I-1:35) and thus cannot use any techniques.

Theme 3. Online materials not in use

Participants talked about not utilizing online materials because they 'did not remember they existed' (I-2:19), some said that they use similar materials from other sources like 
'Instagram where they are easy to find' (I-2:2) and that 'the materials have the same exercises that I already know' (I-2:2).

\section{Individual differences between theme distributions}

Intended and unintended themes were not distributed equally between individuals, instead, responses 'clustered' in different engager types (File S3). The participants whose comments did not indicate acceptance of the LMI gave limited or negative commentary on most intervention components. They reported choosing not to enact the skills even though enactment might increase their ability to do PA. Overall, only three of the interviewed participants were labelled as 'negative engagers'.

Almost all interviewed participants provided commentary under mixed skill reception and many under unintended enactment. Interestingly, there were participants who described enacting the skills as intended but still paradoxically either failed to realize it or just refused the idea of self-regulation. For example, the participant who described optimal enactment of self-regulation techniques later said that 'detailed planning does not suit me' and 'I can't do a precise plan on what I will do, where I do, when I do it'. Hence, most of the participants could be characterized as 'ambivalent engagers'.

The participants who had the most indications of 'intended engagement' seemed to be personally affected by noticing that they have poor physical health. They discussed their motivation to change their health for the better, for example, because they looked 'too overweight', had to 'catch breath often', or were 'embarrassed by sweating easily'. They also have had good PA experiences, recently or in their past. They all mentioned that they can choose their own ways to be active. These three participants could be labelled 'positive engagers'.

\section{Discussion}

We investigated how Let's Move It intervention participants describe (1) intervention acceptability, (2) receipt, and (3) enactment of skills (BCTs). Indicating high acceptability, remarks on intervention were mainly positive and related to affective attitude, perceived effectiveness, and intervention coherence, as only a small minority viewed the sessions boring or uninformative. No theme related to ethicality, burden or opportunity costs. In terms of receipt, analysis indicated understanding of the main ideas, and how and why to perform the skills delivered in LMI. Problems in receipt included framing intervention merely as a school course and skills not attributed useful for the participants themselves. Arguably, the 'not for me' commentary could reflect poor fit with the participants' value system but as the commentary focused on specific intervention content, it was considered to reflect receipt rather than acceptability (Figure 1). As for skill enactment, participants rarely enacted the self-motivation and self-regulatory techniques outside the intervention settings. For those who reported enactment, apparent misunderstandings included, for example, non-specific planning instead of specific plans, and outcome-related goals instead of behavioural goals. Here too, the 'planning and self-monitoring require time and effort' commentary could reflect the burden and opportunity costs dimensions of acceptability, but as the comments regard skill use in the target setting, it is conceptualized as enactment.

The analysis revealed between-person differences in the valence of comments. The within-individual analysis revealed that those who expressed low acceptance of the LMI 
programme gave negative comments on the intervention components as well and that the participants with the highest intended receipt and enactment seem to have been emotionally affected by noticing their poor health and had positive experiences of PA in their past. Understanding how PA is related to one's personal well-being has previously been identified as triggering agentic PA increase in youth (Kostamo, Vesala, \& Hankonen, 2019).

Retaining acceptability as a distinguishable construct enabled pinpointing the engagement issues to receipt and enactment, as there seemed to be a discrepancy between understanding the usefulness of a certain skill in general and perceiving it useful for oneself. That is, despite high acceptability, participants did not always enact the skills. It is possible that the participants understood enactment of skills differently than intended, that is, only in a time-consuming form, and did not perceive simple plans or setting a PA date as planning. Also, perceiving interventions strictly as school courses may limit skill enactment in participants' everyday lives. Indeed, some participants talk about their reluctance to plan due to it reminding them of school or feeling forced to do it. It is important to consider how school-based interventions could be constructed in a way that participants perceive the content more applicable across all life domains where also other motivations are at play. Furthermore, it is worth noting that some adolescents might find having a friend as a prerequisite for PA.

The aspect of engagement is important for intervention design: Identifying and addressing possible 'weak links' in advance may enhance efficacy, optimally in feasibility studies. Despite comprehensive feasibility testing and optimization of the LMI (Hankonen et al., 2017), these analyses still indicate partial failure to illustrate the usefulness of selfregulation techniques for students' everyday life. It would be interesting if more studies systematically compared whether optimization needs identified in the feasibility phase and the measures undertaken to amend them lead to substantial improvements in the RCT phase. It should be noted that the aim here was not to make generalizations (Smith, 2018), but to analyse participants' interpretations that are central in inducing change.

As a limitation, inherent in interview studies, is a possible social desirability bias. Interviewers were trained to avoid this by explicitly stressing the value of both negative and positive views and confidentiality to the participants. Second, it is possible that some elements are more prevalent than others due to directly asking about some intervention content (e.g., PA planning), thus prompting participants to talk about those, whereas other intervention-related content was induced by the participants (e.g., main messages). A more inductive approach might have resulted in more variety, but even a data-driven analysis cannot be completed without any analytic preconceptions (Braun \& Clarke, 2006). Abductive approach offers balance: A more inductive approach would not have been informed by the concepts of acceptability, receipt, and enactment, and a more deductive approach could have resulted in challenges in identifying a theory of engagement to form specific research questions and to analyse the data.

Despite calls to assess intervention fidelity (Bellg et al., 2004), very few health behaviour change interventions focus on the participant side (Rixon et al., 2016). Key limitations of existing assessments include, for example, that they often confuse terms such as receipt, satisfaction, and acceptability and are poorly reported precluding replication and interpretation. Our study has attempted to make clear conceptual distinctions of key concepts of intervention engagement and demonstrate the value of qualitative assessments within effectiveness trials.

In conclusion, this study makes a methodological contribution to the field in demonstrating a novel way to assess engagement in interventions, as well as addressing 
some of the methodological gaps of existing research. In addition, it demonstrates the processes and patterns in participants' engagement with the LMI intervention. Here, despite high acceptability, we identified a gap between receipt and enactment of skills mostly due to the perception of planning being burdensome, which might have led to limited changes in PA levels (Hankonen et al., submitted). Hence, optimization efforts could be guided towards reframing the self-regulatory skills more clearly as fit for anyone and less time-consuming, and underpinning the automation of their use in daily life.

\section{Acknowledgements}

This work was supported by the Ministry of Education and Culture [Grant Number OKM/81/ 626/2014] and Academy of Finland [Grant Numbers 285283 and 304114]. We would like to thank the teacher and student participants, intervention facilitators and research interviewers, and collaborators.

\section{Conflicts of interest}

All authors declare no conflict of interest.

\section{Author contributions}

Minttu Palsola: Conceptualization, Data curation, Formal analysis, Investigation, Methodology, Visualization, Writing - original draft, Writing - review \& editing. Elina Renko: Conceptualization, Investigation, Methodology, Writing - review \& editing. Katri Kostamo: Conceptualization, Data curation, Investigation, Methodology, Validation, Writing review \& editing. Fabiana Lorencatto: Conceptualization, Investigation, Writing - review \& editing. Nelli Hankonen: Conceptualization, Funding acquisition, Investigation, Methodology, Project administration, Resources, Supervision, Writing - review \& editing.

\section{Data availability statement}

Data is available on request from the authors and later from the Finnish Social Science Data Archive.

\section{References}

Bellg, A. J., Borrelli, B., Resnick, B., Hecht, J., Minicucci, D. S., Ory, M., .. Czajkowski, S. (2004). Enhancing treatment fidelity in health behavior change studies: Best practices and recommendations from the NIH behavior change consortium. Health Psychology, 23, 443451. https://doi.org/10.1037/0278-6133.23.5.443

Braun, V., \& Clarke, V. (2006). Using thematic analysis in psychology. Qualitative Research in Psychology, 3(2), 77-101. https://doi.org/10.1191/1478088706qp063oa

Carver, C. S., \& Scheier, M. F. (1982). Control theory: A useful conceptual framework for personality-social, clinical, and health psychology. Psychological Bulletin, 92(1), 111-135. https://doi.org/10.1037/0033-2909.92.1.111

Deaton, A., \& Cartwright, N. (2018). Reflections on randomized control trials. Social Science \& Medicine, 210, 86-90. https://doi.org/10.1016/j.socscimed.2018.04.046 
Deci, E. L., \& Ryan, R. M. (2000). The "what" and "why" of goal pursuits: Human needs and the selfdetermination of behavior. Psychological Inquiry, 11(4), 227-268. https://doi.org/10.1207/ S15327965PLI1104_01

Elgar, F. J., Pförtner, T.-K., Moor, I., De Clercq, B., Stevens, G. W. J. M., \& Currie, C. (2015). Socioeconomic inequalities in adolescent health 2002-2010: A time-series analysis of 34 countries participating in the Health Behaviour in School-aged Children study. The Lancet, 385 , 2088-2095. https://doi.org/10.1016/S0140-6736(14)61460-4

Fishbein, M., \& Ajzen, I. (2010). Predicting and changing behavior: The reasoned action approach. New York, NY: Psychology Press.

Hankonen, N., Absetz, P., \& Araújo-Soares, V. (in press). Changing activity behaviors in vocational school students: The stepwise development of the 'Let's Move it' intervention. Health Psychology and Behavioral Medicine. (Under review).

Hankonen, N., Haukkala, A., Palsola, M., Heino, M. T. J., Tokola, K., Sund, R. ... Vasankari, T. (2019). Effectiveness of the Let's Move It Multilevel School-Based Intervention on Physical Activity and Sedentary Behavior: A Cluster Randomised Clinical Trial. Manuscript submitted for publication. (submitted).

Hankonen, N., Heino, M. T. J., Araújo-Soares, V., Sniehotta, F. F., Sund, R., Vasankari, T., . . Haukkala, A. (2016). 'Let's Move It' - A school-based multilevel intervention to increase physical activity and reduce sedentary behaviour among older adolescents in vocational secondary schools: A study protocol for a cluster-randomised trial. BMC Public Health, 16, 451. https://doi.org/10. 1186/s12889-016-3094-x

Hankonen, N., Heino, M. T. J., Hynynen, S.-T., Laine, H., Araújo-Soares, V., Sniehotta, F. F., ... Haukkala, A. (2017). Randomised controlled feasibility study of a school-based multi-level intervention to increase physical activity and decrease sedentary behaviour among vocational school students. International Journal of Behavioral Nutrition and Physical Activity, 14(1), 37. https://doi.org/10.1186/s12966-017-0484-0

Heino, M. T. J., Knittle, K., Fried, E., Sund, R., Haukkala, A., Borodulin, K., . . Hankonen, N. (2019). Visualisation and network analysis of physical activity and its determinants: Demonstrating opportunities in analysing baseline associations in the Let's Move It trial. Health Psychology and Behavioral Medicine, 7(1), 269-289. https://doi.org/10.1080/21642850.2019.1646136

Hynynen, S.-T., van Stralen, M. M., Sniehotta, F. F., Araújo-Soares, V., Hardeman, W., Chinapaw, M. J. M., ... Hankonen, N. (2016). A systematic review of school-based interventions targeting physical activity and sedentary behaviour among older adolescents. International Review of Sport and Exercise Psychology, 9(1), 22-44. https://doi.org/10.1080/1750984X.2015. 1081706

Kostamo, K., Jallinoja, P., Vesala, K. M., Araújo-Soares, V., Sniehotta, F. F., \& Hankonen, N. (2019). Using the critical incident technique for qualitative process evaluation of interventions: The example of the "Let's Move It" trial. Social Science \& Medicine, 232, 389-397. https://doi.org/ 10.1016/j.socscimed.2019.05.014

Kostamo, K., Vesala, K. M., Hankonen, N. (2019). What triggers changes in adolescents' physical activity? Analysis of critical incidents during childhood and youth in student writings. Psychology of Sport and Exercise, 45, 101564. http://doi.org/10.1016/j.psychsport.2019. 101564

Michie, S., Richardson, M., Johnston, M., Abraham, C., Francis, J., Hardeman, W., ... Wood, C. E. (2013). The behavior change technique taxonomy (v1) of 93 hierarchically clustered techniques: Building an international consensus for the reporting of behavior change interventions. Annals of Behavioral Medicine, 46(1), 81-95. https://doi.org/10.1007/ s12160-013-9486-6

Moore, G. F., Audrey, S., Barker, M., Bond, L., Bonell, C., Hardeman, W., . . Baird, J. (2015). Process evaluation of complex interventions: Medical research council guidance. BMJ, 350, h1258. https://doi.org/10.1136/bmj.h1258

Patton, M. Q. (1990). Qualitative evaluation and research methods. Thousand Oaks, CA: Sage Publications Inc. 
Rixon, L., Baron, J., McGale, N., Lorencatto, F., Francis, J., \& Davies, A. (2016). Methods used to address fidelity of receipt in health intervention research: A citation analysis and systematic review. BMC Health Services Research, 16, 663. https://doi.org/10.1186/s12913-016-1904-6

Sekhon, M., Cartwright, M., \& Francis, J. (2017). Acceptability of healthcare interventions: An overview of reviews and development of a theoretical framework. BMC Health Services Research, 17(1), 88. https://doi.org/10.1186/s12913-017-2031-8

Smith, B. (2018). Generalizability in qualitative research: Misunderstandings, opportunities and recommendations for the sport and exercise sciences. Qualitative Research in Sport, Exercise and Health, 10(1), 137-149. https://doi.org/10.1080/2159676X.2017.1393221

Tavory, I., \& Timmermans, S. (2014). Abductive analysis: Theorizing qualitative research. Chicago, IL: University of Chicago Press.

Tuomi, J., \& Sarajärvi, A. (2002). Laadullinen tutkimus ja sisällönanalyysi [Qualitative research and content analysis]. Helsinki, Finland: Tammi.

Walton, H., Spector, A., Tombor, I., \& Michie, S. (2017). Measures of fidelity of delivery of, and engagement with, complex, face-to-face health behaviour change interventions: A systematic review of measure quality. British Journal of Health Psychology, 22, 872-903. https://doi.org/ 10.1111/bjhp.12260

Received 18 September 2019; revised version received 28 April 2020

\section{Supporting Information}

The following supporting information may be found in the online edition of the article:

File S1. Steps and decisions needed to carry out an interview study using thematic analysis to investigate acceptability, receipt, and enactment in interventions.

File S2. General risks and pitfalls in abductive approach interview research and how they were managed.

File S3. Participants grouped by the valence of their comments under each main theme.

File S4. I-1 Interview guide.

File S5. I-2 Interview guide. 